\title{
Total Oxidant and Antioxidant Status in Patients with Obstructive Sleep Apnea and the Effect of Continuous Positive Airway Pressure
}

\author{
Hyeon Hui Kang, In Kyoung Kim, and Sang Haak Lee \\ Division of Pulmonary, Critical Care and Sleep Medicine, Department of Internal Medicine, Eunpyeong St. Mary's Hospital, \\ College of Medicine, The Catholic University of Korea, Seoul, Korea
}

\begin{abstract}
Objective: We explored total oxidant status (TOS) and total antioxidant status (TAS) in patients with obstructive sleep apnea (OSA) and compared them between patients and control group. We also evaluated the effect of continuous positive airway pressure (CPAP) treatment on oxidative stress parameters. Methods: A total of 150 consecutive patients admitted to the sleep clinic for OSA evaluation between 2009 and 2013 were enrolled in the study, including 119 OSA patients and 31 simple snoring control subjects, confirmed by polysomnography. In both groups, serum TAS and TOS levels were measured. TAS and TOS levels of 16 patients in the OSA group were evaluated after CPAP titration. Results: The OSA group consisted of 35 mild, 29 moderate, and 56 severe OSA patients. There was no association between the severity of sleep apnea and parameters of oxidative stress. No significant difference was found in serum TAS and TOS levels of the OSA group and the controls or before and after CPAP titration in 16 OSA patients. Conclusion: In this study, we found no differences in oxidative stress parameters between control and OSA groups. A single night of CPAP treatment does not seem to influence the oxidant-antioxidant status in OSA.

Key Words: Sleep apnea; Obstructive; Oxidative stress; Continuous positive airway pressure; Oxidants; Antioxidants

Received: September 10, 2019 Revised: September 19, 2019 Accepted: September 19, 2019

Corresponding author: Hyeon Hui Kang, MD, PhD, Division of Pulmonary, Critical Care and Sleep Medicine, Department of Internal Medicine, Eunpyeong St. Mary's Hospital, College of Medicine, The Catholic University of Korea, 1021 Tongil-ro, Eunpyeong-gu, Seoul 03312, Korea.

Tel: 82-2-2030-4339, Fax: 82-2-2030-2617, E-mail: khh3822@catholic.ac.kr

(c) This is an Open Access article distributed under the terms of the Creative Commons Attribution Non-Commercial License (https://creativecommons.org/licenses/bync/4.0) which permits unrestricted non-commercial use, distribution, and reproduction in any medium, provided the original work is properly cited.
\end{abstract}

\section{INTRODUCTION}

Obstructive sleep apnea (OSA) is a common disorder of middle-aged adults, affecting $4 \%$ of men and $2 \%$ of women [1]. The incidence of cardiovascular mortalities and morbidities such as hypertension, ischemic heart disease, arrhythmias, and stroke is higher in patients with OSA than in the general population [2]. OSA is characterized by recurrent upper airway obstruction. $\mathrm{Pa}-$ tients with OSA experience intermittent hypoxia, leading to hypoxemia, hypercapnia, and increased sympathetic activation. Several studies have reported increased oxidative stress in patients with OSA [3-5]. Changes in blood oxygen saturation levels during sleep in OSA may result in the oxidant-antioxidant imbalance in favor of higher oxidants, depending on recurrent hypoxic events [6]. Increased generation of reactive oxygen species in vivo can lead to the depletion of one or more antioxidants, and the loss can be measured as an index of oxidative stress [7]. However, there are several arguments on oxidative stress and OSA.

In this study, we evaluated total oxidant status (TOS) and total antioxidant status (TAS) in patients with OSA and compared their levels between patient and control groups with regard to the demographics and severity of OSA. We also evaluated the effect of continuous positive airway pressure (CPAP) treatment on oxidative stress parameters.

\section{METHODS}

\section{Study participants}

We analyzed the data for 150 consecutive patients who were admitted to the sleep clinic at St. Paul's Hospital, College of Medicine, The Catholic University of Korea, for evaluation of OSA, between 2009 and 2013. The patients had at least one of the following symptoms: snoring, witnessed apnea, excessive daytime sleepiness, and nocturnal choking, and were suspected of having 
OSA. This study and the protocol were approved by Institutional Review Board of St. Paul's Hospital, and written informed consent was obtained from all participants.

The patients underwent blood testing after overnight polysomnography. In OSA groups, 16 patients were readmitted for another overnight polysomnography under CPAP titration. Blood for oxidative stress analysis was drawn around the same time as the first polysomnography.

\section{Polysomnography}

An overnight polysomnographic evaluation with a Somnostar Pro 7-3a (Cardinal Health, Inc., Dublin, OH, USA) was performed in all the patients. The procedure composed of polygraphic recordings from surface electrodes for electroencephalography, electrooculography, electrocardiography, and electromyography and from nasal pressure transducer with thermistors for nasal and oral airflow, tracheal sounds, and thoracic and abdominal respiration. Transcutaneous oxygen saturation was monitored continuously with a finger pulse oximeter. Positional changes during sleep were recorded, and full-night video recordings were also performed during the test period. All of the evaluations were terminated after final waking of the subject in the morning. The data was collected in a computerized polysomnographic system, and the scoring process was performed manually. Sleep was defined according to the criteria of Rechtschaffen and Kales [8]. Respiratory events were scored according to the American Academy of Sleep Medicine criteria [9]. Apnea was defined as a cessation of airflow lasting at least $10 \mathrm{~s}$. A $\geq 30 \%$ reduction of airflow measured using a nasal pressure transducer, lasting for $\geq 10 \mathrm{~s}$ along with $\geq 4 \%$ oxygen desaturation, or decrease in airflow by $50 \%$ or more and lasting at least $10 \mathrm{~s}$ along with a discernible decrease of $3 \%$ or more oxygen saturation, or an electroencephalography arousal was defined as hypopnea. The apnea-hypopnea index (AHI) was defined as the number of apnea and hypopnea events that occurred per hour of sleep. The AHI, when associated with typical symptoms, was scored as follows: AHI $\geq 5$ events/h was diagnosed as OSA. Of these, $15 \geq \mathrm{AHI} \geq 5$ events/ $\mathrm{h}$ was considered mild, $30 \geq \mathrm{AHI}>15$ events/h was considered moderate, and $>30$ events/h was considered severe OSA. Those who had an AHI less than 5 were included in the control group.

\section{Measurement of the total oxidant-antioxidant status}

The serum TAS levels were measured by an automated method [10] using Rel Assay (Rel Assay ${ }^{\circledR}$, Diagnostics kits, Mega Tip, Ga- ziantep, Turkey). In this method, the antioxidant molecules in the sample decolorize the 2,2-azinobis (3-ethylbenzothiazoline-6-sulphonic acid) (ABTS) cationic radical. The decolorization rate is proportional to the amount of the antioxidant molecule present. Trolox, a vitamin E analog, was used as a calibrator. The data was expressed as mmol Trolox equivalents/L. TOS measurement was performed using the Erel method [11]. In this method, oxidants present in the sample oxidize the ferrousion-o-dianisidine complex to ferric ion, and glycerol molecules that are abundantly present in the reaction medium enhance the oxidation reaction. The ferric ion produces a complex color with xylenol orange in an acidic medium. The intensity of the color is related to the number of oxidant molecules present in the sample. The assay was calibrated with hydrogen peroxide, and the results were expressed in terms of the micromolar hydrogen peroxide equivalent, per liter (mmol $\mathrm{H}_{2} \mathrm{O}_{2}$ equivalent/L).

\section{Statistical analysis}

Statistical analysis was performed with SPSS for Windows (18.0, SPSS Inc., Chicago, IL, USA) package software. Numerical variables were summarized with mean \pm standard deviation. Baseline data was compared using one-way analysis of variance. Paired ttest was used for comparing the oxidants and antioxidants before and after polysomnography with CPAP titration. Unpaired t-test was used for comparing parameters between OSA groups and the control group. A value of $\mathrm{p}<0.05$ was considered significant for all statistical analysis.

\section{RESULTS}

A total of 119 subjects were diagnosed with OSA (mild-35; moderate-29; severe-55), and 31 subjects in the simple snoring category were included as control group. The mean age of the OSA subjects was $49.2 \pm 13.6$ years, and that of the control group was $44.81 \pm 13.70$ years with $113(74.8 \%)$ of them were male. However, there was no significant difference in either age or gender among the patients with OSA and the control group. The demographics of the study groups are presented in Table 1 . The mean values of TAS were similar among the OSA groups compared to that of control group $(1.77 \pm 0.13,1.65 \pm 0.45$, respectively) and there was no significant difference between TOS levels of OSA and control group (4.19 $\pm 5.36,4.11 \pm 2.69$, respectively) either. In patients with CPAP titration, the mean age of the subjects was $52.1 \pm 16.4$ years, and the mean value of AHI was 57.6 \pm 26.7 . After CPAP applica-

Table 1. Demographic data, oxidant and antioxidant status of control and OSA groups

\begin{tabular}{lccccc} 
& Control $(\mathrm{n}=31)$ & Mild OSA $(\mathrm{n}=35)$ & Moderate OSA $(\mathrm{n}=29)$ & Severe OSA $(\mathrm{n}=55)$ & $\mathrm{p}$ value \\
Age $($ years $)$ & $44.81 \pm 13.70$ & $47.83 \pm 13.99$ & $51.03 \pm 11.12$ & $49.11 \pm 14.53$ & 0.33 \\
BMI $\left(\mathrm{kg} / \mathrm{m}^{2}\right)$ & $23.64 \pm 3.12$ & $25.26 \pm 3.87$ & $26.42 \pm 4.03$ & $28.63 \pm 4.15$ & $<0.001$ \\
AHI & $2.07 \pm 1.49$ & $9.00 \pm 2.62$ & $23.73 \pm 3.86$ & $60.51 \pm 21.80$ & $1.77 \pm 0.12$ \\
TAS & $1.65 \pm 0.45$ & $1.80 \pm 0.10$ & $1.75 \pm 0.16$ & 3.001 \\
TOS & $4.11 \pm 2.69$ & $4.20 \pm 3.49$ & $5.62 \pm 9.67$ & 0.06 \\
\hline
\end{tabular}

OSA: obstructive sleep apnea, BMI: body mass index, AHI: apnea-hypopnea index, TAS: total antioxidant status, TOS: total oxidant status 
Table 2. Comparisons of serum TAS and TOS levels after CPAP therapy

\begin{tabular}{cccc}
$\mathrm{n}=16$ & Before CPAP & After CPAP & $\mathrm{p}$ value \\
TAS, $\mathrm{mmol}$ Trolox Eq/L & $1.75 \pm 0.11$ & $1.78 \pm 0.11$ & 0.43 \\
TOS, $\mu \mathrm{mol} \mathrm{H}_{2} \mathrm{O}_{2} \mathrm{Eq} / \mathrm{L}$ & $6.54 \pm 12.57$ & $3.01 \pm 1.67$ & 0.31 \\
\hline
\end{tabular}

TAS: total antioxidant status, TOS: total oxidant status, CPAP: continuous positive airway pressure

tion, an increase in the TAS levels from $1.75 \pm 0.11$ to $1.78 \pm 0.11$ was noted, while TOS levels decreased from $6.54 \pm 12.57$ to $3.01 \pm 1.67$ but were statistically insignificant (Table 2).

\section{DISCUSSION}

In this study, we compared TOS and TAS levels between OSA patients and simple snoring control group. However, no significant difference was found in oxidant and antioxidative status between the OSA and control groups. We investigated the effects of CPAP on antioxidative status to find that the serum TOS and TAS levels were not significantly different even after CPAP application.

Oxidative stress has been reported to be involved in the pathogenesis of various diseases, such as hyperlipidemia, diabetes, and hypertension [12]. The relationship between oxidant-antioxidant status and OSA has been investigated in previous studies; the current knowledge on increased oxidative stress in the patient with OSA is controversial. Schulz et al. [3] demonstrated enhanced neutrophil superoxide release in OSAS patients compared with controls, which was reversed by CPAP therapy. Barceló et al. [13] reported that patients with OSA had lower TAS, vitamin A, and vitamin $\mathrm{E}$ levels. In contrast, some authors have failed to show increased oxidative stress, either by finding no differences in lipid peroxidation [14-17] or in circulation free nitrotyrosine [18]. In recent studies, Svatikova et al. [15] found that heathy OSAS patients, without any other co-morbidities, did not show evidence of higher oxidative stress, as assessed by measuring plasma lipid peroxidation products before sleep and after $4 \mathrm{~h}$ of untreated OSAS. Katsoulis et al. [19] excluded patients with comorbid diseases. In that study, the impact of the severity of disease of TAS and the effect of overnight CPAP administration on TAS were analyzed, but no statistically significant changes in TAS were observed in patients with severe OSA. In our study, we did not find a correlation between oxidative stress and CPAP application. The same limitation may explain the absence of influence of a few hours of CPAP treatment on oxidant-antioxidant status.

These conflicting results may be explained by several factors including the presence of co-morbidities and medications in patients with OSA, both of which can have significant effects on oxidative stress measurement; the absence of controls matched for body mass index and obesity (as obesity may cause oxidative stress independent of OSA) and finally, the timing of oxidative stress measurements. It is important to differentiate between any acute effect of hypoxemia resulting from an apneic event during sleep and any chronic state of heightened oxidative stress that may be sustained in OSA patients even during the daytime [15]. Although we excluded the subjects having a history of chronic and acute systemic infection at the time of the study, we did not exclude subjects having chronic inflammatory disease, hypertension, ischemic cardiovascular disease and hyperlipidemia. In the current study, TOS levels were lowest in patients with severe sleep apnea, and TAS levels were higher in patients with severe sleep apnea compared with moderate sleep apnea. The possibility of medication or treatment affecting the oxidative stress in severe OSA patients cannot be ruled out.

The effect of CPAP therapy in oxidative stress is unclear. Svatikova et al. earlier reported that CPAP therapy did not elicit any change in antioxidant enzymes, and in the absence of significant co-morbidities, sleep apnea did not in itself, initiate the generation of oxidative stress. However, it is possible that in the setting of co-morbidities such as hypertension, vascular disease and the metabolic syndrome, the oxidative consequence of sleep apnea may become apparent [15]. In this study, mean TOS levels, in OSA patients, after CPAP application seem to be influenced from TOS values before CPAP (from 6.54 to 3.01), they were not statistically significant. In terms of the impact of CPAP on the oxidative stress, our study is limited by the small sample size, and therefore, future studies with larger sample cohorts are warranted.

In conclusion, the present study shows that OSA is not related with the level of TAS and TOS suggesting an increased oxidative stress. One night of CPAP treatment does not seem to impact the antioxidant status. Further studies are required on the impact of oxidative stress and the effect of long-term CPAP therapy in patients with OSA.

\section{Conflicts of Interest}

The authors have no potential conflicts of interest to disclose.

\section{Author Contributions}

Conceptualization: Hyeon Hui Kang. Formal analysis: Hyeon Hui Kang. Investigation: In Kyoung Kim. Methodology: Hyeon Hui Kang. Project administration: Sang Haak Lee. Resources: Sang Haak Lee. Writing_original draft: Hyeon Hui Kang. Writing_review \& editing: Hyeon Hui Kang.

\section{ORCID iD}

Hyeon Hui Kang (1)

https://orcid.org/0000-0001-5860-9407

\section{REFERENCES}

1. Young T, Palta M, Dempsey J, Skatrud J, Weber S, Badr S. The occurrence of sleep-disordered breathing among middle-aged adults. N Engl J Med 1993; 328:1230-1235.

2. Somers VK, White DP, Amin R, Abraham WT, Costa F, Culebras A, et al. Sleep apnea and cardiovascular disease: an American Heart Association/ american College Of Cardiology Foundation Scientific Statement from the American Heart Association Council for High Blood Pressure Research Professional Education Committee, Council on Clinical Cardiology, Stroke Council, and Council On Cardiovascular Nursing. In collaboration with the National Heart, Lung, and Blood Institute National Center on Sleep Disorders Research (National Institutes of Health). Circulation 2008;118:1080 
1111.

3. Schulz R, Mahmoudi S, Hattar K, Sibelius U, Olschewski H, Mayer K, et al. Enhanced release of superoxide from polymorphonuclear neutrophils in obstructive sleep apnea. Impact of continuous positive airway pressure therapy. Am J Respir Crit Care Med 2000;162:566-570.

4. Dyugovskaya L, Lavie P, Lavie L. Increased adhesion molecules expression and production of reactive oxygen species in leukocytes of sleep apnea patients. Am J Respir Crit Care Med 2002;165:934-939.

5. Christou K, Kostikas K, Pastaka C, Tanou K, Antoniadou I, Gourgoulianis KI. Nasal continuous positive airway pressure treatment reduces systemic oxidative stress in patients with severe obstructive sleep apnea syndrome. Sleep Med 2009;10:87-94.

6. Wysocka E, Cofta S, Cymerys M, Gozdzik J, Torlinski L, Batura-Gabryel H. The impact of the sleep apnea syndrome on oxidant-antioxidant balance in the blood of overweight and obese patients. J Physiol Pharmacol 2008;59 Suppl 6:761-769.

7. Hallliwell B, Gutteridge JMC. Detection of free radicals and other reactive species: trapping and fingerprinting. In: Haliwell B, Gutteridge JMC, editors. Free radicals in biology and medicine. 3rd ed. Oxford: Oxford University Press, 1998, p. 351-429.

8. Rechtschaffen A, Kales A. A manual of standardized terminology, techniques and scoring system for sleep stages in human subjects. Los Angeles, CA: Brain information Service, Brain Research Institute, University of California; 1968.

9. Iber C, Ancoli-Israel S, Chesson A, Quan SF; American Academy of Sleep Medicine. The AASM manual for the scoring of sleep and associated events: rules, terminology and technical specifications. 1st ed. Westchester, IL: American Academy of Sleep Medicine; 2007.

10. Erel O. A novel automated direct measurement method for total antioxidant capacity using a new generation, more stable ABTS radical cation. Clin Biochem 2004;37:277-285.

11. Erel O. A novel automated method to measure total antioxidant response against potent free radical reactions. Clin Biochem 2004;37:112-119.

12. Yang S, Zhu H, Li Y, Lin H, Gabrielson K, Trush MA, et al. Mitochondrial adaptations to obesity-related oxidant stress. Arch Biochem Biophys 2000; 378:259-268.

13. Barceló A, Barbé F, de la Peña M, Vila M, Pérez G, Piérola J, et al. Antioxidant status in patients with sleep apnoea and impact of continuous positive airway pressure treatment. Eur Respir J 2006;27:756-760.

14. Oztürk L, Mansour B, Yüksel M, Yalçin AS, Celikoğlu F, Gökhan N. Lipid peroxidation and osmotic fragility of red blood cells in sleep-apnea patients. Clin Chim Acta 2003;332:83-88.

15. Svatikova A, Wolk R, Lerman LO, Juncos LA, Greene EL, McConnell JP, et al. Oxidative stress in obstructive sleep apnoea. Eur Heart J;26:2435-2439.

16. Wali SO, Bahammam AS, Massaeli H, Pierce GN, Iliskovic N, Singal PK, et al. Susceptibility of LDL to oxidative stress in obstructive sleep apnea. Sleep 1998;21:290-296.

17. Alzoghaibi MA, Bahammam AS. Lipid peroxides, superoxide dismutase and circulating IL-8 and GCP-2 in patients with severe obstructive sleep apnea: a pilot study. Sleep Breath 2005;9:119-126.

18. Svatikova A, Wolk R, Wang HH, Otto ME, Bybee KA, Singh RJ, et al. Circulating free nitrotyrosine in obstructive sleep apnea. Am J Physiol Regul Integr Comp Physiol 2004;287:R284-R287.

19. Katsoulis K, Kontakiotis T, Spanogiannis D, Vlachogiannis E, Kougioulis M, Gerou S, et al. Total antioxidant status in patients with obstructive sleep apnea without comorbidities: the role of the severity of the disease. Sleep Breath 2011;15:861-866. 